\title{
Boundedness character of a fourth-order system of difference equations
}

\author{
Stevo Stević ${ }^{1,2^{*}}$, Bratislav Iričanin ${ }^{3}$ and Zdeněk Šmarda ${ }^{4,5}$
}

"Correspondence: sstevic@ptt.rs 'Mathematical Institute of the Serbian Academy of Sciences, Knez Mihailova 36/III, Beograd, 11000, Serbia

${ }^{2}$ Operator Theory and Applications Research Group, Department of Mathematics, King Abdulaziz University, P.O. Box 80203, Jeddah, 21589, Saudi Arabia

Full list of author information is available at the end of the article

\author{
Abstract \\ The boundedness character of positive solutions of the following system of difference \\ equations: $x_{n+1}=A+\frac{y_{n}^{p}}{x_{n-3}^{r}}, y_{n+1}=A+\frac{x_{n}^{p}}{y_{n-3}^{n}}, n \in \mathbb{N}_{0}$, when $\min \{A, r\}>0$ and $p \geq 0$, is \\ studied. \\ MSC: Primary 39A10; 39A20 \\ Keywords: system of difference equations; bounded solutions; unbounded \\ solutions; positive solutions
}

\section{Introduction}

Concrete nonlinear difference equations and systems, especially those which are not closely related to differential ones, have attracted a lot of attention recently (see, for example, [1-33] and the references therein). Among them, symmetric and close to symmetric systems of difference equations, whose study was essentially initiated by Papaschinopoulos and Schinas in the mid-1990s, have attracted a considerable interest (see, for example, [4, 7-11, 13, 14, 22-32]). For example, in [7] Papaschinopoulos and Schinas studied the oscillatory behavior, the boundedness character, and the global stability of positive solutions of the following close to symmetric system of difference equations:

$$
x_{n+1}=A+\frac{y_{n}}{x_{n-p}}, \quad y_{n+1}=A+\frac{x_{n}}{y_{n-q}}, \quad n \in \mathbb{N}_{0},
$$

where $A>0$ and $p, q \in \mathbb{N}$. It should be noted that the system is rational. On the other hand, for the case $p=q$ the system obviously becomes symmetric, that is, it is of the following form:

$$
x_{n}=f\left(x_{n-k}, y_{n-l}\right), \quad y_{n}=f\left(y_{n-k}, x_{n-l}\right), \quad n \in \mathbb{N}_{0},
$$

for some $k, l \in \mathbb{N}$.

On the other hand, a systematic study of positive solutions of nonlinear difference equations containing non-integer powers of their dependent variables began by Stević et al., approximately since the publication of [15], where the first nontrivial results related to the

(c) 2015 Stević et al. This article is distributed under the terms of the Creative Commons Attribution 4.0 International License (http://creativecommons.org/licenses/by/4.0/), which permits unrestricted use, distribution, and reproduction in any medium, provided you give appropriate credit to the original author(s) and the source, provide a link to the Creative Commons license, and indicate if changes were made. 
following difference equation were given:

$$
x_{n+1}=\alpha+\frac{x_{n-1}^{p}}{x_{n}^{p}}, \quad n \in \mathbb{N}_{0}
$$

where $\min \{\alpha, p\}>0$.

A good prototype including (1) is the following difference equation:

$$
x_{n}=\alpha+\frac{x_{n-k}^{p}}{x_{n-l}^{r}}, \quad n \in \mathbb{N}_{0},
$$

where $k, l \in \mathbb{N}, k \neq l, \min \{\alpha, r\}>0$, and $p \geq 0$, which was proposed for studying by Stević at numerous talks. Some special cases of this, the corresponding max-type difference equation or related equations has been studied considerably (see, for example, $[1,2,5,12,16-21$, 30, 31] and the references therein).

Motivated by these two lines of investigations Stević has proposed recently studying symmetric and close to symmetric systems of difference equations which, among others, stem from special cases of (2).

Motivated by all above mentioned work, and especially by [19], here we investigate the boundedness character of the solutions of the next system of difference equations

$$
x_{n+1}=A+\frac{y_{n}^{p}}{x_{n-3}^{r}}, \quad y_{n+1}=A+\frac{x_{n}^{p}}{y_{n-3}^{r}}, \quad n \in \mathbb{N}_{0} \text {, }
$$

when $\min \{A, r\}>0, p \geq 0$, and $x_{-i}, y_{-i}>0, i \in\{0,1,2,3\}$. Our results extend and complement some results in [19].

By using the induction and the equations in (3) we see that if $x_{-i}, y_{-i}>0, i \in\{0,1,2,3\}$, then

$$
\min \left\{x_{n}, y_{n}\right\}>0, \quad n \geq-3
$$

which means that positive initial values generate positive solutions of system (3). Moreover, we have

$$
\min \left\{x_{n}, y_{n}\right\}>A, \quad n \in \mathbb{N}
$$

The case $p=0$ is simple. Namely, in this case by using (4) into (3) is obtained

$$
\max \left\{x_{n+1}, y_{n+1}\right\}<A+\frac{1}{A^{r}}, \quad n \geq 4,
$$

which means that all positive solutions of system (3) in this case are bounded. In fact, since

$$
A<\min \left\{x_{n}, y_{n}\right\} \leq \max \left\{x_{n}, y_{n}\right\}<A+\frac{1}{A^{r}}, \quad n \geq 5
$$

they are persistent.

For a solution $\left(x_{n}, y_{n}\right)_{n \geq-3}$ of system (3) it is said that it is unbounded if

$$
\sup _{n \geq-3}\left\|\left(x_{n}, y_{n}\right)\right\|_{\mathbb{R}^{2}}=\sup _{n \geq-3} \sqrt{x_{n}^{2}+y_{n}^{2}}=+\infty \text {. }
$$


Otherwise, the solution is bounded, that is, if there is a nonnegative constant $M$ such that

$$
\sup _{n \geq-3}\left\|\left(x_{n}, y_{n}\right)\right\|_{\mathbb{R}^{2}} \leq M<+\infty
$$

\section{Main results}

In this section we prove the main results in this paper, all of which are related to the boundedness character, that is, the boundedness of all positive solutions of system (3) or the existence of an unbounded solution of the system depending on the values of parameters $A$, $p$, and $r$.

Theorem 1 Assume that $\min \{A, p, r\}>0$ and $27 p^{4}<256 r$. Then all positive solutions of system (3) are bounded.

Proof Using the equations in (3), we have

$$
\begin{aligned}
& x_{n+1}=A+\frac{y_{n}^{p}}{x_{n-3}^{r}} \\
& =A+\left(\frac{y_{n}}{x_{n-3}^{\frac{r}{p}}}\right)^{p} \\
& =A+\left(\frac{A}{x_{n-3}^{\frac{r}{p}}}+\frac{x_{n-1}^{p}}{x_{n-3}^{\frac{r}{p}} y_{n-4}^{r}}\right)^{p} \\
& =A+\left(\frac{A}{x_{n-3}^{\frac{r}{p}}}+\left(\frac{x_{n-1}}{x_{n-3}^{\frac{r}{p^{2}}} y_{n-4}^{\frac{r}{p}}}\right)^{p}\right)^{p} \\
& =A+\left(\frac{A}{x_{n-3}^{\frac{r}{p}}}+\left(\frac{A}{x_{n-3}^{\frac{r}{p^{2}}} y_{n-4}^{\frac{r}{p}}}+\frac{y_{n-2}^{p}}{x_{n-3}^{\frac{r}{p^{2}}} y_{n-4}^{\frac{r}{p}} x_{n-5}^{r}}\right)^{p}\right)^{p} \\
& =A+\left(\frac{A}{x_{n-3}^{\frac{r}{p}}}+\left(\frac{A}{x_{n-3}^{\frac{r}{p^{2}}} y_{n-4}^{\frac{r}{p}}}+\left(\frac{y_{n-2}}{x_{n-3}^{\frac{r}{p^{3}}} y_{n-4}^{\frac{r}{p^{2}}} x_{n-5}^{\frac{r}{p}}}\right)^{p}\right)^{p}\right)^{p} \\
& =A+\left(\frac{A}{x_{n-3}^{\frac{r}{p}}}+\left(\frac{A}{x_{n-3}^{\frac{r}{p^{2}}} y_{n-4}^{\frac{r}{p}}}+\left(\frac{A}{x_{n-3}^{\frac{r}{p^{3}}} y_{n-4}^{\frac{r}{p^{2}}} x_{n-5}^{\frac{r}{p}}}+\frac{x_{n-3}^{p-\frac{r}{p^{3}}}}{y_{n-4}^{\frac{r}{p^{2}}} x_{n-5}^{\frac{r}{p}} y_{n-6}^{r}}\right)^{p}\right)^{p}\right)^{p} \\
& =A+\left(\frac{A}{x_{n-3}^{\frac{r}{p}}}+\left(\frac{A}{x_{n-3}^{\frac{r}{p^{2}}} y_{n-4}^{\frac{r}{p}}}+\left(\frac{A}{x_{n-3}^{\frac{r}{p^{3}}} y_{n-4}^{\frac{r}{p^{2}}} x_{n-5}^{\frac{r}{p}}}\right.\right.\right. \\
& \left.\left.\left.+\left(\frac{x_{n-3}}{y_{n-4}^{\frac{r}{p^{2}} /\left(p-\frac{r}{p^{3}}\right)} x_{n-5}^{\frac{r}{p} /\left(p-\frac{r}{p^{3}}\right)} y_{n-6}^{r /\left(p-\frac{r}{p^{3}}\right)}}\right)^{p-\frac{r}{p^{3}}}\right)^{p}\right)^{p}\right)^{p} \\
& =A+\left(\frac{A}{x_{n-3}^{\frac{r}{p}}}+\left(\frac{A}{x_{n-3}^{\frac{r}{p^{2}}} y_{n-4}^{\frac{r}{p}}}+\left(\frac{A}{x_{n-3}^{\frac{r}{p^{3}}} y_{n-4}^{\frac{r}{p^{2}}} x_{n-5}^{\frac{r}{p}}}+\left(\frac{x_{n-3}}{y_{n-4}^{a_{1}} x_{n-5}^{b_{1}} y_{n-6}^{c_{1}}}\right)^{p-\frac{r}{p^{3}}}\right)^{p}\right)^{p}\right)^{p} \text {, }
\end{aligned}
$$

where

$$
a_{1}:=\frac{r}{p^{2}} /\left(p-\frac{r}{p^{3}}\right), \quad b_{1}=\frac{r}{p} /\left(p-\frac{r}{p^{3}}\right), \quad c_{1}:=r /\left(p-\frac{r}{p^{3}}\right) .
$$


Now using the first equation in (3) in (6) we get

$$
\begin{aligned}
x_{n+1}= & A+\left(\frac{A}{x_{n-3}^{\frac{r}{p}}}+\left(\frac{A}{x_{n-3}^{\frac{r}{p^{2}}} y_{n-4}^{\frac{r}{p}}}+\left(\cdots+\left(\frac{A}{y_{n-4}^{a_{1}} x_{n-5}^{b_{1}} y_{n-6}^{c_{1}}}+\frac{y_{n-4}^{p-a_{1}}}{x_{n-5}^{b_{1}} y_{n-6}^{c_{1}} x_{n-7}^{r}}\right)^{p-\frac{r}{p^{3}}}\right)^{p}\right)^{p}\right)^{p} \\
= & A+\left(\frac{A}{x_{n-3}^{\frac{r}{p}}}+\left(\frac{A}{x_{n-3}^{\frac{r}{p^{2}}} y_{n-4}^{\frac{r}{p}}}+\left(\cdots+\left(\frac{A}{y_{n-4}^{a_{1}} x_{n-5}^{b_{1}} y_{n-6}^{c_{1}}}\right.\right.\right.\right. \\
& \left.\left.\left.\left.+\left(\frac{y_{n-4}}{x_{n-5}^{\frac{b_{1}}{p-a_{1}}} y_{n-6}^{\frac{c_{1}}{p-a_{1}}} x_{n-7}^{\frac{r}{p-a_{1}}}}\right)^{p-a_{1}}\right)^{p-\frac{r}{p^{3}}}\right)^{p}\right)^{p}\right)^{p} \\
= & A+\left(\frac{A}{x_{n-3}^{\frac{r}{p}}+\left(\frac{A}{x_{n-3}^{\frac{r}{p^{2}}} y_{n-4}^{\frac{r}{p}}}+\left(\cdots+\left(\frac{A}{y_{n-4}^{a_{1}} x_{n-5}^{b_{1}} y_{n-6}^{c_{1}}}\right.\right.\right.}\right. \\
& \left.\left.\left.+\left(\frac{y_{n-4}}{x_{n-5}^{a_{2}} y_{n-6}^{b_{2}} x_{n-7}^{c_{2}}}\right)^{p-a_{1}}\right)^{p-\frac{r}{p^{3}}}\right)^{p}\right)^{p},
\end{aligned}
$$

where

$$
a_{2}:=\frac{b_{1}}{p-a_{1}}, \quad b_{2}=\frac{c_{1}}{p-a_{1}}, \quad c_{2}:=\frac{r}{p-a_{1}} .
$$

Assume that for some $k \geq 2$ we have proved that the following equalities hold:

$$
\begin{aligned}
x_{n+1} & =A+\left(\frac{A}{x_{n-3}^{\frac{r}{p}}}+\cdots\left(\frac{x_{n-2 k+1}}{y_{n-2 k}^{a_{2 k-3}} x_{n-2 k-1}^{b_{2 k-3}} y_{n-2 k-2}^{c_{2 k-3}}}\right)^{p-a_{2 k-4}} \cdots\right)^{p} \\
& =A+\left(\frac{A}{x_{n-3}^{\frac{r}{p}}}+\cdots\left(\frac{y_{n-2 k}}{x_{n-2 k-1}^{a_{2 k-2}} y_{n-2 k-2}^{b_{2 k-2}} x_{n-2 k-3}^{c_{2 k-2}}}\right)^{p-a_{2 k-3}} \cdots\right)^{p},
\end{aligned}
$$

where the sequences $a_{k}, b_{k}$, and $c_{k}$ are defined by

$$
a_{k+1}=\frac{b_{k}}{p-a_{k}}, \quad b_{k+1}=\frac{c_{k}}{p-a_{k}}, \quad c_{k+1}=\frac{r}{p-a_{k}},
$$

with

$$
a_{0}=\frac{r}{p^{3}}, \quad b_{0}=\frac{r}{p^{2}}, \quad c_{0}=\frac{r}{p} .
$$

Using again the equations in (3) and the recurrent relations in (10), we have

$$
\begin{aligned}
x_{n+1}= & A+\left(\frac{A}{x_{n-3}^{\frac{r}{p}}}+\cdots\left(\frac{y_{n-2 k}}{x_{n-2 k-1}^{a_{2 k-2}} y_{n-2 k-2}^{b_{2 k-2}} x_{n-2 k-3}^{c_{2 k-2}}}\right)^{p-a_{2 k-3}} \cdots\right)^{p} \\
= & A+\left(\frac{A}{x_{n-3}^{\frac{r}{p}}+\cdots\left(\frac{A}{x_{n-2 k-1}^{a_{2 k-2}} y_{n-2 k-2}^{b_{2 k-2}} x_{n-2 k-3}^{c_{2 k-2}}}\right.}\right. \\
& \left.\left.+\frac{x_{n-2 k-1}^{p-a_{2 k-2}}}{y_{n-2 k-2}^{b_{2 k-2}} x_{n-2 k-3}^{c_{2 k-2}} y_{n-2 k-4}^{r}}\right)^{p-a_{2 k-3}} \cdots\right)^{p}
\end{aligned}
$$




$$
\begin{aligned}
& =A+\left(\frac{A}{x_{n-3}^{\frac{r}{p}}}+\cdots\left(\frac{A}{x_{n-2 k-1}^{a_{2 k-2}} y_{n-2 k-2}^{b_{2 k-2}} x_{n-2 k-3}^{c_{2 k-2}}}\right.\right. \\
& \left.\left.+\left(\frac{x_{n-2 k-1}}{y_{n-2 k-2}^{\frac{b_{2 k-2}}{p-a_{2 k-2}}} x_{n-2 k-3}^{\frac{c_{2 k-2}}{p-a_{2 k-2}}} y_{n-2 k-4}^{\frac{r}{p-a_{2 k-2}}}}\right)^{p-a_{2 k-2}}\right)^{p-a_{2 k-3}} \cdots\right)^{p} \\
& =A+\left(\frac{A}{x_{n-3}^{\frac{r}{p}}}+\cdots\left(\frac{A}{x_{n-2 k-1}^{a_{2 k-2}} y_{n-2 k-2}^{b_{2 k-2}} x_{n-2 k-3}^{c_{2 k-2}}}\right.\right. \\
& \left.\left.+\left(\frac{x_{n-2 k-1}}{y_{n-2 k-2}^{a_{2 k-1}} x_{n-2 k-3}^{b_{2 k-1}} y_{n-2 k-4}^{c_{2 k-1}}}\right)^{p-a_{2 k-2}}\right)^{p-a_{2 k-3}} \cdots\right)^{p} \\
& x_{n+1}=A+\left(\frac{A}{x_{n-3}^{\frac{r}{p}}}+\cdots\left(\frac{A}{y_{n-2 k-2}^{a_{2 k-1}} x_{n-2 k-3}^{b_{2 k-1}} y_{n-2 k-4}^{c_{2 k-1}}}\right.\right. \\
& \left.\left.+\frac{y_{n-2 k-2}^{p-a_{2 k-1}}}{x_{n-2 k-3}^{b_{2 k-1}} y_{n-2 k-4}^{c_{2 k-1}} x_{n-2 k-5}^{r}}\right)^{p-a_{2 k-2}} \cdots\right)^{p} \\
& =A+\left(\frac{A}{x_{n-3}^{\frac{r}{p}}}+\cdots\left(\frac{y_{n-2 k-2}}{x_{n-2 k-3}^{\frac{b_{2 k-1}}{p-a_{2 k-1}}} y_{n-2 k-4}^{\frac{c_{2 k-1}}{p-a_{2 k-1}}} x_{n-2 k-5}^{\frac{r}{p-a_{2 k-1}}}}\right)^{p-a_{2 k-1}} \cdots\right)^{p} \\
& =A+\left(\frac{A}{x_{n-3}^{\frac{r}{p}}}+\cdots\left(\frac{y_{n-2 k-2}}{x_{n-2 k-3}^{a_{2 k}} y_{n-2 k-4}^{b_{2 k}} x_{n-2 k-5}^{c_{2 k}}}\right)^{p-a_{2 k-1}} \cdots\right)^{p} \text {. }
\end{aligned}
$$

From (6), (7), (12), (14), and the method of induction it follows that (8) and (9) hold for every $k \geq 2$, and for every $n \geq 2 k$.

If $p^{4}>r$, then

$$
\begin{aligned}
& a_{0}=\frac{r}{p^{3}}<\frac{r}{p^{2}} /\left(p-\frac{r}{p^{3}}\right)=a_{1}, \\
& b_{0}=r / p^{2}<\frac{r}{p} /\left(p-\frac{r}{p^{3}}\right)=b_{1}, \\
& c_{0}=r / p<r /\left(p-\frac{r}{p^{3}}\right)=c_{1} .
\end{aligned}
$$

From this and by using recurrent relations (10), it follows that $a_{k}, b_{k}$, and $c_{k}$ increase, as far as $a_{k}<p$. On the other hand, (10) implies

$$
a_{k+1}=\frac{r}{\left(p-a_{k}\right)\left(p-a_{k-1}\right)\left(p-a_{k-2}\right)}, \quad k \geq 2 .
$$

Hence, if $a_{k}<p$ for every $k \in \mathbb{N}$ we see that there is a finite $\operatorname{limit}_{k \rightarrow \infty} a_{k}=x^{*} \in(0, p]$, and that $x^{*}$ is a solution of the equation

$$
f(x)=x(p-x)^{3}-r=0 .
$$

We have $f(0)=f(p)=-r$ and $f^{\prime}(x)=(x-p)^{2}(p-4 x)$. Hence $\max _{x \in[0, p]} f(x)=f(p / 4)$. Since by a condition of the theorem

$$
f(p / 4)=\frac{27 p^{4}}{256}-r<0,
$$

we arrive at a contradiction. 
This guarantees the existence of the smallest $l \in \mathbb{N}$ such that $a_{l-1}<p$ and $a_{l} \geq p$. This, along with (13) with $l=2 k_{0}-1$, implies that

$$
\begin{aligned}
& x_{n+1}=A+\left(\frac{A}{x_{n-3}^{\frac{r}{p}}}+\cdots\left(\frac{A}{y_{n-2 k_{0}-2}^{a_{2 k_{0}-1} x_{n-2 k_{0}-3}^{b_{2 k}-1} y_{n-2 k_{0}-4}^{c_{2 k_{0}-1}}}}\right.\right. \\
& \left.\left.+\frac{y_{n-2 k_{0}-2}^{p-a_{2 k_{0}-1}}}{x_{n-2 k_{0}-3} y_{n-2 k_{0}-4} x_{n-2 k_{0}-5}^{r}}\right)^{p-a_{2 k_{0}-2}} \ldots\right)^{p} \\
& \leq A+\left(\frac{1}{A^{\frac{r}{p}-1}}+\cdots\left(\frac{1}{A^{a_{2 k_{0}-1}+b_{2 k_{0}-1}+c_{2 k_{0}-1}-1}}\right.\right. \\
& \left.\left.+\frac{1}{A^{a_{2 k_{0}-1}+b_{2 k_{0}-1}+c_{2 k_{0}-1}+r-p}}\right)^{p-a_{2 k_{0}-2}} \cdots\right)^{p},
\end{aligned}
$$

for $n \geq 2 k_{0}+6$, which implies the boundedness of $x_{n}$ in this case. Due to the symmetry of system (3) the boundedness of $y_{n}$ follows and consequently the boundedness of the solution. If $l=2 k_{0}-2$, then from (11) it follows that

$$
\begin{aligned}
& x_{n+1}=A+\left(\frac{A}{x_{n-3}^{\frac{r}{p}}}+\cdots\left(\frac{A}{x_{n-2 k_{0}-1} y_{n-2 k_{0}-2} x_{n-2 k_{0}-3}}\right.\right. \\
& \left.\left.+\frac{x_{n-2 k_{0}-1}^{p-a_{2 k_{0}-2}}}{y_{n-2 k_{0}-2}^{b_{2 k_{0}-2} x_{2 k_{0}-2} y_{n}-3} y_{n-2 k_{0}-4}^{r}}\right)^{p-a_{2 k_{0}-3}} \cdots\right)^{p} \\
& \leq A+\left(\frac{1}{A^{\frac{r}{p}-1}}+\cdots\left(\frac{1}{A^{a_{2 k_{0}-2}+b_{2 k_{0}-2}+c_{2 k_{0}-2^{-1}}}}\right.\right. \\
& \left.\left.+\frac{1}{A^{a_{2 k_{0}-2}+b_{2 k_{0}-2}+c_{2 k_{0}-2}+r-p}}\right)^{p-a_{2 k_{0}-3}} \cdots\right)^{p} \text {, }
\end{aligned}
$$

for $n \geq 2 k_{0}+5$, which implies the boundedness of $x_{n}$ in this case.

Due to the symmetry of system (3) we also have

$$
y_{n+1} \leq A+\left(\frac{1}{A^{\frac{r}{p}-1}}+\cdots\left(\frac{1}{A^{a_{2 k_{0}-2}+b_{2 k_{0}-2}+c_{2 k_{0}-2}-1}}+\frac{1}{A^{a_{2 k_{0}-2}+b_{2 k_{0}-2}+c_{2 k_{0}-2}+r-p}}\right)^{p-a_{2 k_{0}-3}} \cdots\right)^{p},
$$

for $n \geq 2 k_{0}+5$, which along with the previous inequality implies the boundedness of the solution.

If $p^{4} \leq r$, then using (4) in (5) we get

$$
\begin{aligned}
x_{n+1} & =A+\left(\frac{A}{x_{n-3}^{\frac{r}{p}}}+\left(\frac{A}{x_{n-3}^{\frac{r}{p^{2}}} y_{n-4}^{\frac{r}{p}}}+\left(\frac{A}{x_{n-3}^{\frac{r}{p^{3}}} y_{n-4}^{\frac{r}{p^{2}}} x_{n-5}^{\frac{r}{p}}}+\frac{x_{n-3}^{p-\frac{r}{p^{3}}}}{y_{n-4}^{\frac{r}{p^{2}}} x_{n-5}^{\frac{r}{p}} y_{n-6}^{r}}\right)^{p}\right)^{p}\right)^{p} \\
& \leq A+\left(\frac{1}{A^{\frac{r}{p}-1}}+\left(\frac{1}{A^{\frac{r}{p^{2}}+\frac{r}{p}-1}}+\left(\frac{1}{A^{\frac{r}{p^{3}}+\frac{r}{p^{2}}+\frac{r}{p}-1}}+\frac{1}{A^{\frac{r}{p^{3}}+\frac{r}{p^{2}}+\frac{r}{p}+r-p}}\right)^{p}\right)^{p}\right)^{p},
\end{aligned}
$$

for $n \geq 7$, from which the boundedness follows in the case.

Due to the symmetry of system (3) we see that the inequality

$$
y_{n+1} \leq A+\left(\frac{1}{A^{\frac{r}{p}-1}}+\left(\frac{1}{A^{\frac{r}{p^{2}}+\frac{r}{p}-1}}+\left(\frac{1}{A^{\frac{r}{p^{3}}+\frac{r}{p^{2}}+\frac{r}{p}-1}}+\frac{1}{A^{\frac{r}{p^{3}}+\frac{r}{p^{2}}+\frac{r}{p}+r-p}}\right)^{p}\right)^{p}\right)^{p}
$$


holds for $n \geq 7$, from which along with the previous inequality the boundedness of the solution follows.

Remark 1 Note that if $a_{k}=p$ for some $k \in \mathbb{N}$, then $a_{k+1}, b_{k+1}$, and $c_{k+1}$ are not defined. However, if this happens then above mentioned index $l$ is chosen to be this $k$. For such chosen $l$ is obtained an upper bound for positive solutions of system (3) in the way described in the proof of Theorem 1.

Theorem 2 Assume that $\min \{A, p, r\}>0,27 p^{4} \geq 256 r$, and $p \geq 4 / 3$ (where at least one of these two inequalities is strict), or $r<p-1<1 / 3$. Then system (3) has positive unbounded solutions.

Proof Assume that $\left(x_{n}, y_{n}\right)_{n \geq-3}$ is a positive solution of (3). Then we have

$$
\begin{aligned}
& x_{n+1} \geq \frac{y_{n}^{p}}{x_{n-3}^{r}}, \\
& y_{n+1} \geq \frac{x_{n}^{p}}{y_{n-3}^{r}},
\end{aligned}
$$

for $n \in \mathbb{N}_{0}$.

Let

$$
z_{n}=\ln \left(x_{n} y_{n}\right), \quad n \geq-3
$$

Taking the logarithm of the both sides in (15), (16), then summing such obtained inequalities, it follows that

$$
z_{n+1}-p z_{n}+r z_{n-3} \geq 0, \quad n \in \mathbb{N}_{0} .
$$

Let

$$
P(\lambda)=\lambda^{4}-p \lambda^{3}+r
$$

Then $P(0)=r$ and

$$
P^{\prime}(\lambda)=\lambda^{2}(4 \lambda-3 p)
$$

from which it follows that the polynomial $P(\lambda)$ has a local minimum at $\lambda=3 p / 4$, and according to the conditions of the theorem

$$
P(3 p / 4)=-27 p^{4} / 256+r \leq 0 .
$$

If $p>4 / 3$, then $3 p / 4>1$. From this, (19) and since

$$
\lim _{\lambda \rightarrow+\infty} P(\lambda)=+\infty,
$$

it follows that there is $\lambda_{1}>1$ such that $P\left(\lambda_{1}\right)=0$. If $p=4 / 3$, inequality (19) is strict, $3 p / 4=1$, and since (20) holds, we also see that there is $\lambda_{1}>1$ such that $P\left(\lambda_{1}\right)=0$. 
Now assume that $r<p-1<1 / 3$. Then $P(1)=1-p+r<0$ and since (20) holds, we again see that there is $\lambda_{1}>1$ such that $P\left(\lambda_{1}\right)=0$.

Let

$$
P_{2}(\lambda)=P(\lambda) /\left(\lambda-\lambda_{1}\right)=\lambda^{3}+a \lambda^{2}+b \lambda+c
$$

and

$$
u_{n}=z_{n}+a z_{n-1}+b z_{n-2}+c z_{n-3} .
$$

Then inequality (17) can be written in the following form:

$$
u_{n+1}-\lambda_{1} u_{n} \geq 0
$$

Choose $x_{-i}, y_{-i}, i \in\{0,1,2,3\}$, such that $u_{0}>0$. For example, to get $u_{0}>0$, it is enough to choose $x_{-i}, y_{-i}, i \in\{0,1,2,3\}$, such that

$$
x_{0} y_{0}>|a|\left|x_{-1} y_{-1}\right|+|b|\left|x_{-2} y_{-2}\right|+|c|\left|x_{-3} y_{-3}\right| \text {. }
$$

From this and (21) it follows that

$$
u_{n} \geq \lambda_{1}^{n} u_{0}
$$

Since $u_{0}>0$ and $\lambda_{1}>1$, by letting $n \rightarrow \infty$ in (22) we obtain $u_{n} \rightarrow+\infty$ as $n \rightarrow \infty$. If $z_{n}$ were bounded then $u_{n}$ would be also bounded, which would be a contradiction. Hence $z_{n}$ is unbounded. From this and since

$$
\sqrt{x_{n}^{2}+y_{n}^{2}} \geq 2 x_{n} y_{n}=2 e_{n}^{z}, \quad n \in \mathbb{N}_{0}
$$

we have

$$
\sup _{n \geq-3} \sqrt{x_{n}^{2}+y_{n}^{2}}=+\infty
$$

that is, the solution of system (3) is unbounded, completing the proof of the theorem.

Theorem 3 Assume that $\min \{A, p, r\}>0$ and $p=r+1$. Then system (3) has positive unbounded solutions.

Proof Let

$$
x_{0} y_{0}>x_{-1} y_{-1}>x_{-2} y_{-2}>x_{-3} y_{-3}>0 \text {. }
$$

Since $p=r+1$, system (3) is

$$
x_{n+1}=A+\frac{y_{n}^{r+1}}{x_{n-3}^{r}}, \quad y_{n+1}=A+\frac{x_{n}^{r+1}}{y_{n-3}^{r}}, \quad n \in \mathbb{N}_{0} \text {. }
$$


Multiplying these two equations we easily obtain

$$
\frac{x_{n+1} y_{n+1}}{x_{n} y_{n}}>\left(\frac{x_{n} y_{n}}{x_{n-3} y_{n-3}}\right)^{r}
$$

from which with $n=0$, it follows that

$$
\frac{x_{1} y_{1}}{x_{0} y_{0}}>\left(\frac{x_{0} y_{0}}{x_{-3} y_{-3}}\right)^{r}>1
$$

Assume that we have proved

$$
x_{k} y_{k}>x_{k-1} y_{k-1}, \quad \text { for }-2 \leq k \leq n \text {. }
$$

Then from (25) and (26) we have

$$
\frac{x_{n+1} y_{n+1}}{x_{n} y_{n}}>\left(\frac{x_{n} y_{n}}{x_{n-3} y_{n-3}}\right)^{r}>1, \quad n \in \mathbb{N}_{0}
$$

Hence

$$
x_{n+1} y_{n+1}>x_{n} y_{n},
$$

for every $n \geq-3$. If $x_{n} y_{n}$ was bounded, then there would be a finite positive $\lim _{n \rightarrow \infty} x_{n} y_{n}=c$. Letting $n \rightarrow \infty$ in the product of equations in (24) we would obtain $c \geq A^{2}+c$, which would be a contradiction. Hence, all the solutions of (24) satisfying (23) are unbounded.

Theorem 4 Assume that $\min \{A, r\}>0$ and $p \in(0,1)$. Then every positive solution of system (3) is bounded.

Proof Since $x_{n}>A, n \in \mathbb{N}$, we have

$$
x_{n+1} \leq A+\frac{y_{n}^{p}}{A^{r}}, \quad y_{n+1} \leq A+\frac{x_{n}^{p}}{A^{r}},
$$

for $n \geq 4$, where $\left(x_{n}, y_{n}\right)_{n \geq-3}$ is an arbitrary positive solution of system (3).

Hence

$$
x_{n+1}+y_{n+1} \leq 2 A+\frac{2\left(x_{n}+y_{n}\right)^{p}}{A^{r}},
$$

for $n \geq 4$.

Let $\left(z_{n}\right)_{n \geq 4}$ be the solution of the equation

$$
z_{n+1}=2 A+\frac{2 z_{n}^{p}}{A^{r}}, \quad n \geq 4,
$$

such that $z_{4}=x_{4}+y_{4}$.

Since

$$
f(x)=2 A+\frac{2 x^{p}}{A^{r}}
$$


is increasing on $\mathbb{R}_{+}$, a simple inductive argument shows that

$$
x_{n}+y_{n} \leq z_{n}, \quad \text { for } n \geq 4 \text {. }
$$

Since $p \in(0,1)$ function $f$ is concave, which implies that there is a unique fixed point $x^{*}$ of $f$ and that the next condition

$$
(f(x)-x)\left(x-x^{*}\right)<0, \quad x \in(0, \infty) \backslash\left\{x^{*}\right\}
$$

holds.

If $z_{4} \in\left(0, x^{*}\right]$ condition (31) implies that $\left(z_{n}\right)_{n \geq 4}$ is nondecreasing and bounded above by $x^{*}$, and if $z_{4} \geq x^{*}$ that it is nonincreasing and bounded below by $x^{*}$. Hence $\left(z_{n}\right)_{n \geq 4}$ is bounded, which along with (30) implies the boundedness of $\left(x_{n}\right)_{n \geq 4}$ and $\left(y_{n}\right)_{n \geq 4}$, from which the result easily follows.

In the next theorem we use the fact that the comparison equation is a linear first order difference equation, which is solvable in closed form. For recent application of this and related equations see, for example, [4, 22, 23, 25-29, 33].

Theorem 5 Assume that $p=1, r>0$, and $A>\sqrt[r]{2}$. Then every positive solution of system (3) is bounded.

Proof From the proof of Theorem 4 we see that any positive solution $\left(x_{n}, y_{n}\right)_{n \geq-3}$ of system (3) satisfies (28) with $p=1$.

Let $\left(z_{n}\right)_{n \geq 4}$ be the solution of the equation

$$
z_{n+1}=2 A+\frac{2 z_{n}}{A^{r}}, \quad n \geq 4
$$

such that $z_{4}=x_{4}+y_{4}$. Then clearly (30) also holds.

It is well known that (32) is solvable. Using its solution in closed form is easily proved that

$$
\lim _{n \rightarrow \infty} z_{n}=\frac{2 A^{r+1}}{A^{r}-2}
$$

from which the boundedness of $\left(z_{n}\right)_{n \geq 4}$ follows. This fact along with (30) implies the boundedness of $\left(x_{n}\right)_{n \geq 4}$ and $\left(y_{n}\right)_{n \geq 4}$, from which the result easily follows.

Remark 2 The boundedness character of positive solutions of system (3) in the following two cases:

(a) $r \leq 27 p^{4} / 256,1<p<r+1, r<1 / 3$;

(b) $r \leq 27 p^{4} / 256, p=1, A \in(0, \sqrt[r]{2}]$,

is not known to us. Hence, we leave the cases to the interested reader.

The authors declare that they have no competing interests. 


\section{Author details}

'Mathematical Institute of the Serbian Academy of Sciences, Knez Mihailova 36/III, Beograd, 11000, Serbia. ${ }^{2}$ Operator Theory and Applications Research Group, Department of Mathematics, King Abdulaziz University, P.O. Box 80203, Jeddah, 21589, Saudi Arabia. ${ }^{3}$ Faculty of Electrical Engineering, Belgrade University, Bulevar Kralja Aleksandra 73, Beograd, 11000, Serbia. ${ }^{4}$ CEITEC - Central European Institute of Technology, Brno University of Technology, Technická 3058/10, Brno, 616 00, Czech Republic. ${ }^{5}$ Department of Mathematics, FEEC - Faculty of Electrical Engineering and Communication, Brno University of Technology, Technická 3058/10, Brno, 616 00, Czech Republic.

\section{Acknowledgements}

The work of Stevo Stević is supported by the Serbian Ministry of Education and Science projects III 41025 and III 44006. The work of Bratislav Iričanin is supported by the Serbian Ministry of Education and Science projects III 41025 and Ol 171007. The work of Zdeněk Šmarda was realized in CEITEC - Central European Institute of Technology with research infrastructure supported by the project CZ.1.05/1.1.00/02.0068 financed from European Regional Development Fund. He was also supported by the project FEKT-S-14-2200 of Brno University of Technology.

\section{Received: 27 July 2015 Accepted: 23 September 2015 Published online: 13 October 2015}

\section{References}

1. Berenhaut, K, Foley, J, Stević, S: The global attractivity of the rational difference equation $y_{n}=1+\left(y_{n-k} / y_{n-m}\right)$. Proc. Am. Math. Soc. 135(1), 1133-1140 (2007)

2. Berenhaut, K, Stević, S: The behaviour of the positive solutions of the difference equation $x_{n}=A+\left(x_{n-2} / x_{n-1}\right)^{p}$. J. Differ. Equ. Appl. 12(9), 909-918 (2006)

3. Berezansky, L, Braverman, E: On impulsive Beverton-Holt difference equations and their applications. J. Differ. Equ. Appl. 10(9), 851-868 (2004)

4. Berg, L, Stević, S: On some systems of difference equations. Appl. Math. Comput. 218, 1713-1718 (2011)

5. Iričanin, B, Stević, S: On a class of third-order nonlinear difference equations. Appl. Math. Comput. 213, 479-483 (2009)

6. Karakostas, GL: Asymptotic 2-periodic difference equations with diagonally self-invertible responses. J. Differ. Equ. Appl. 6, 329-335 (2000)

7. Papaschinopoulos, G, Schinas, CJ: On a system of two nonlinear difference equations. J. Math. Anal. Appl. 219(2), 415-426 (1998)

8. Papaschinopoulos, G, Schinas, CJ: On the behavior of the solutions of a system of two nonlinear difference equations. Commun. Appl. Nonlinear Anal. 5(2), 47-59 (1998)

9. Papaschinopoulos, G, Schinas, CJ: Invariants for systems of two nonlinear difference equations. Differ. Equ. Dyn. Syst. 7(2), 181-196 (1999)

10. Papaschinopoulos, G, Schinas, CJ: Invariants and oscillation for systems of two nonlinear difference equations. Nonlinear Anal. TMA 46(7), 967-978 (2001)

11. Papaschinopoulos, G, Schinas, CJ: On the dynamics of two exponential type systems of difference equations. Comput. Math. Appl. 64(7), 2326-2334 (2012)

12. Papaschinopoulos, G, Schinas, CJ, Stefanidou, G: On the nonautonomous difference equation $x_{n+1}=A_{n}+\left(x_{n-1}^{p} / x_{n}^{q}\right)$. Appl. Math. Comput. 217, 5573-5580 (2011)

13. Stefanidou, G, Papaschinopoulos, G, Schinas, C: On a system of max difference equations. Dyn. Contin. Discrete Impuls. Syst., Ser. A 14(6), 885-903 (2007)

14. Stefanidou, G, Papaschinopoulos, G, Schinas, C: On a system of two exponential type difference equations. Commun. Appl. Nonlinear Anal. 17(2), 1-13 (2010)

15. Stević, S: On the recursive sequence $x_{n+1}=\alpha+\left(x_{n-1}^{p} / x_{n}^{p}\right)$. J. Appl. Math. Comput. 18(1-2), 229-234 (2005)

16. Stević, S: On the recursive sequence $x_{n+1}=A+\left(x_{n}^{p} / x_{n-1}^{r}\right)$. Discrete Dyn. Nat. Soc. 2007, Article ID 40963 (2007)

17. Stević, S: On the recursive sequence $x_{n+1}=\max \left\{c, x_{n}^{p} / x_{n-1}^{p}\right\}$. Appl. Math. Lett. 21(8), 791-796 (2008)

18. Stević, S: Boundedness character of a class of difference equations. Nonlinear Anal. TMA 70, 839-848 (2009)

19. Stević, S: Boundedness character of a fourth order nonlinear difference equation. Chaos Solitons Fractals 40, 2364-2369 (2009)

20. Stević, S: On a generalized max-type difference equation from automatic control theory. Nonlinear Anal. TMA 72 1841-1849 (2010)

21. Stević, S: On a nonlinear generalized max-type difference equation. J. Math. Anal. Appl. 376, 317-328 (2011)

22. Stević, S: On a solvable rational system of difference equations. Appl. Math. Comput. 219, 2896-2908 (2012)

23. Stević, S: On a third-order system of difference equations. Appl. Math. Comput. 218, 7649-7654 (2012)

24. Stević, S: On some periodic systems of max-type difference equations. Appl. Math. Comput. 218, 11483-11487 (2012)

25. Stević, S: Solutions of a max-type system of difference equations. Appl. Math. Comput. 218, $9825-9830$ (2012)

26. Stević, S: Domains of undefinable solutions of some equations and systems of difference equations. Appl. Math. Comput. 219, 11206-11213 (2013)

27. Stević, S: Representation of solutions of bilinear difference equations in terms of generalized Fibonacci sequences. Electron. J. Qual. Theory Differ. Equ. 2014, Article No. 67 (2014)

28. Stević, S, Alghamdi, MA, Alotaibi, A, Elsayed, EM: Solvable product-type system of difference equations of second order. Electron. J. Differ. Equ. 2015, Article No. 169 (2015)

29. Stević, S, Alghamdi, MA, Alotaibi, A, Shahzad, N: On a higher-order system of difference equations. Electron. J. Qual. Theory Differ. Equ. 2013, Article No. 47 (2013)

30. Stević, S, Alghamdi, MA, Alotaibi, A, Shahzad, N: On a nonlinear second order system of difference equations. Appl. Math. Comput. 219, 11388-11394 (2013)

31. Stević, S, Alghamdi, MA, Alotaibi, A, Shahzad, N: Long-term behavior of positive solutions of a system of max-type difference equations. Appl. Math. Comput. 235, 567-574 (2014)

32. Stević, S, Alghamdi, MA, Alotaibi, A, Shahzad, N: Boundedness character of a max-type system of difference equations of second order. Electron. J. Qual. Theory Differ. Equ. 2014, Article No. 45 (2014)

33. Stević, S, Diblík, J, Iričanin, B, Šmarda, Z: Solvability of nonlinear difference equations of fourth order. Electron. J. Differ Equ. 2014, Article No. 264 (2014) 